\title{
COMPUTER SIMULATION OF AUTOMOBILE FIRES
}

Using CFD (Computer Fluid Dynamics) theory and its practical knowledge has become widespread in such academic disciplines as aerodynamics, fluid dynamics, combustion engineering and other fields. However, in disciplines, which examine the ongoing processes in larger sizes, CFD was applied during the last decade only. One of such discipline is a spread of fire. Fire processes are a very complicated and complex phenomenon consisting of combustion, radiation, turbulence, fluid dynamics, pressure and other physical and chemical processes. In the paper, we describe the use of FDS (Fire Dynamics Simulator) for the simulation of automobile/vehicle fires in different environment.

Keywords: computer fire simulation, CFD, fire in tunnel and car park, FDS

\section{Introduction}

In recent years, the number of car fires on roads has been increased gradually in Slovakia. As a result of this tendency, growing number of injured and killed people and damage caused by these fires has been noticed. Reasons of these fires are different and not always related to a technical or electrical failure of cars. Particularly, arson may cause great material damages in overcrowd town agglomeration and built-up areas, or in wildland-urban interface causing wildfire. Fires in road tunnels, although are still relatively rare in Slovakia, can be very destructive and dangerous when they arise. That is why it is necessary to pay considerable attention to fire safety issues of roads and motorway tunnels. Especially, when the traffic prognosis for Slovakia as well as for other EU countries indicates that the quantity of transported goods and number of cars and tunnels will still grow quite rapidly.

A good knowledge about complex phenomena and processes occurring during the fire in different environments is a significant component of fire safety. One of economically least expensive methods how to obtain the knowledge is computer simulation of fire. It is especially valid in the case of car fires in tunnels, because full-scale fire experiments in tunnels could cause serious damages of material and technical equipment in the tunnel. Nowadays, monitoring the processes development in fire environment allows a relatively good knowledge about the dynamics of liquids and gases. Existing software tools provided by CFD simulation also enable to visualize their development. In the scientific literature, computer simulation of fire was firstly formulated in seventies as zonal models and later as multi-zone models [1, 2]. In these models, the fire area is divided into separate fire areas (zones) so that each of these ongoing processes have been settled. The theoretical basis of these methods was the laws of conservation of mass and energy. The whole space was divided into two zones: a warmer upper portion of space containing heat and smoke and a lower part, which was significantly less affected by heat and smoke. However, the uses of these methods were significantly limited.

CFD models were introduced in nineties and reached significant development and relatively widespread use in various fields of human activity. Several advanced systems directed to simulation of combustion processes have been developed. CFX, PHOENICS and SMARTFIRE systems provide alternative radiation models which may offer a good performance. We use FDS (Fire Dynamics Simulator) system, whose first version was developed in 2000 by the NIST (National Institute of Standards and Technology, USA). At present days, significantly better FDS version 5.5 is already available.

During past ten years, number of papers paid attention to numerical simulation of car fires in open or closed spaces. Particularly, they focused on the research of fires in tunnels and garages and their possible consequences. In $[3,4,5,6]$, fires in open or closed garages were described. The effect of ventilation on the fire spread and smoke movement in car park was simulated and an optimal starting time of a smoke extraction system was studied. Other useful results were obtained by a computer simulation of car fires in tunnels. To optimize the regulation of ventilation system for the case of such fires is also very important. Thermal stratification, maximum smoke temperature under the ceiling and smoke backflow were also studied for different types of fires in tunnels $[7,8,9,10]$.

The aim of this paper is to concisely present theoretical foundations of numerical modelling combustion processes implemented in FDS to highlight also the relatively large-scale use of this system

\footnotetext{
* Ladislav Halada ${ }^{1}$, Peter Weisenpacher ${ }^{1}$, Gabriel Oksa ${ }^{2}$, Jan Glasa ${ }^{1}$, Martin Becka ${ }^{2}$

${ }^{1}$ Institute of Informatics, Slovak Academy of Sciences, Bratislava, Slovakia, E-mail: upsyhala@savba.sk

${ }^{2}$ Mathematical Institute, Slovak Academy of Sciences, Bratislava, Slovakia
} 
for various types of car fires. Moreover, we present the recent results of computer simulation of fire in engine compartment. Simulation of this type of car fire has not been published in the literature yet.

\section{Basic equations of the FDS model}

FDS [11] solves a form of conservation equations for low speed, thermally driven flow. Smoke and heat transfer from fires is the main concern of this system, which also includes the thermal radiation, pyrolysis, combustion of pyrolysis products, flame spread and fire suppression by sprinklers. The basic set of the conservation mass, species, momentum and energy equations are as follows [11]:

$$
\begin{aligned}
& \frac{\partial \rho}{\partial t}+\nabla \cdot \rho u=\dot{m}_{b}^{m} \\
& \frac{\partial}{\partial t}\left(\rho Y_{\alpha}\right)+\nabla \cdot \rho Y_{\alpha} u=\nabla \cdot \rho D_{\alpha} \nabla Y_{\alpha}+\dot{m}_{\alpha}^{m}+\dot{m}_{b, \alpha}^{m} \\
& \frac{\partial}{\partial t}(\rho \boldsymbol{u})+\nabla \cdot \rho \boldsymbol{u} \boldsymbol{u}+\nabla p=\rho \boldsymbol{g}+\boldsymbol{f}_{b}+\nabla \cdot \tau_{i j} \\
& \frac{\partial}{\partial t}\left(\rho h_{s}\right)+\nabla \cdot \rho h_{s} \boldsymbol{u}=\frac{D p}{D t}+\dot{q}^{m}-\dot{q}_{b}^{m}-\nabla \cdot \dot{\boldsymbol{q}}^{n}+\varepsilon
\end{aligned}
$$

where $\dot{m}_{b}^{m}=\sum \dot{m}_{b, \alpha}^{m}$ is the production rate of species by evaporating droplets or particles; $\rho$ is the density; $\boldsymbol{u}=(u, v, w)$ is the velocity vector; $Y_{\alpha}, D_{\alpha}$, and $\dot{m}_{b, \alpha}^{m}$ are the mass fraction, the diffusion coefficient and the mass production rate of $\alpha$-th species per unit volume, respectively; $p$ is the pressure; $f_{b}$ is the external force vector; $\tau$ is the viscous stress tensor; hs is the sensible enthalpy; the term $\dot{q}^{m}$ is the heat release rate per unit volume from a chemical reaction and $\dot{q}_{b}^{m}$ is the energy transferred to the evaporating droplets; and the term represents the conductive and radiative heat fluxes. Note that the use of the material derivative $D() / D t=$ $\partial(\mathrm{)} / \partial \mathrm{t}+\boldsymbol{u} \cdot \operatorname{grad}(\mathrm{)})$ holds in the last equation.

Other two equations, the pressure equation and the equation of state,

$$
\nabla^{2} H=-\frac{\partial}{\partial t}(\nabla \cdot \boldsymbol{u})-\nabla \cdot F \text { and } p=\frac{\rho R T}{W}
$$

are added to the previous four equations. The pressure equation is obtained applying the divergence on the momentum equation. In this equation, the value $H$ represents the total pressure divided by the density. $R$ is the universal gas constant, $T$ is temperature and $\mathrm{W}$ is the molecular weight of the gas mixture.

Thus, we have the set of six equations for six unknowns, which are functions of three spatial dimensions and time: the density $\rho$, three components of $\boldsymbol{u}=(u, v, w)$, the temperature $T$ and the pressure $p$. These equations must be simplified in order to filter out sound waves, which are much faster than typical flow speed. The final numerical scheme is an explicit predictor-corrector finite difference scheme, which is second order accurate in space and time.
The flow variables are updated in time using an explicit secondorder Runge-Kutta scheme.

Boundary conditions are prescribed on walls and vents. All input data for simulation are required in the form of a text file in prescribed format, which describes the coordinate system, geometry of domain and its location in given coordinates, mesh resolution obstacles, boundary conditions, material properties and other different simulations parameters. Important limitations of the system is that the domain should be rectilinear, conforming with underlying grid. The domain is filled with rectangular obstructions representing real objects, which can burn, heat up, conduct heat, etc. Simulation outputs include quantities for gas phase (temperature, velocity, species concentration, visibility, pressure, heat release rate per unit volume, etc.), for solid surfaces (temperature, heat flux, burning rate, etc.), as well as global quantities (total heat release rate, mass and energy fluxes through openings, etc.). These outputs are saved during simulation with desired format for visualization and can be visualized by the Smokeview program.

As it is mentioned in [11], the overall computation can either be treated as Direct Numerical Simulation (DNS), in which the dissipative terms are computed directly, or as Large Eddy Simulation (LES), in which large-scale eddies are computed directly and subgrid-scale dissipative processes are modelled. The numerical algorithm is designed so that LES becomes DNS as grid is refined. Description of the numerical schemes used for the solution of all equations is completely described in [11].

The aim of FDS is to solve practical problems in fire protection engineering, as well as to provide a tool to study basic processes in combustion and fire dynamics. In recent years, it has become a widely used and well tested system by various research teams in the world. It is well known that the accuracy of simulated fires highly depends on grid resolution. Therefore, in many papers authors investigate effects of grid size on different fire characteristics, such as the flame height, radiative heat fluxes, temperature distribution, and so on. In papers [12,13], the effect of computational grid size on predicted characteristics of thermal radiation for fire is investigated. It was observed for different grid sizes that predicted flame heights for 20, 30 and $38 \mathrm{~cm}$ pool fires increase with increasing ratio $D^{*} / \Delta d$ (i.e., with decreasing grid size), where $D^{*}$ is the characteristic diameter and $\Delta d$ is the grid size, and would approach to a certain value. In addition, some studies had shown that the radiative heat transfer is one of dominant heat transfer mechanisms associated with such fires. Therefore, the accurate simulation of radiation strongly influences the prediction of fuel burning rate, smoke management flame inhibition effect, etc. However, the accurate prediction of these distributions depends on the choice of numerical treatments that include also the mesh size.

Another serious problem is the computation of total pressure $H$ which fulfills the pressure equation. This equation solved by numerical scheme gives the Poisson equation, which is solved by a fast Fourier transform. The precision of this solution is very important and has significant influence on simulation outputs. 


\section{Computer simulation of automobile engine compartment fire (Audi 80)}

Simulation of automobile engine compartment fire belongs to the most complex FDS simulation problems. To the best of our knowledge, we do not know any published paper addressed this problem. Due to complex geometry of burning space and objects inside the engine compartment, which affect the fire development and then have to be accurately captured, such a simulation requires very fine mesh resolution and therefore significant computational power for calculation. That is why the design of engine compartment geometry and components in the input FDS file is of great importance. In order to properly model the engine compartment as well as all relevant flammable components in its interior, the corresponding input geometry of simulated space was elaborated using available 3D scans and direct measurements of distances and proportions of detected flammable components. The simulation domain includes plastic, rubber and metallic components which influence the direction of fire and smoke spread in time (see Fig. 1). Engine compartment fire simulation is just the situation in which certain geometric features of engine compartment components do not conform to rectangular mesh, and have to be represented in a different way (electrical cables, tubes, hoses, etc.). The shape of plastic tanks and air filter can be captured almost realistically. However, the rubber tube (small thickness and cylindrical shape) has to be represented by a cluster of thin stripes where the total mass of stripes is equal to the mass of the tube itself. Moreover, the surface to volume ratio of the stripes is the same as that of the tube. Both these parameters, which are crucial for heating up and burning of material, were maintained. By this way some other components, such as paper interior of the air filter box, were modelled.

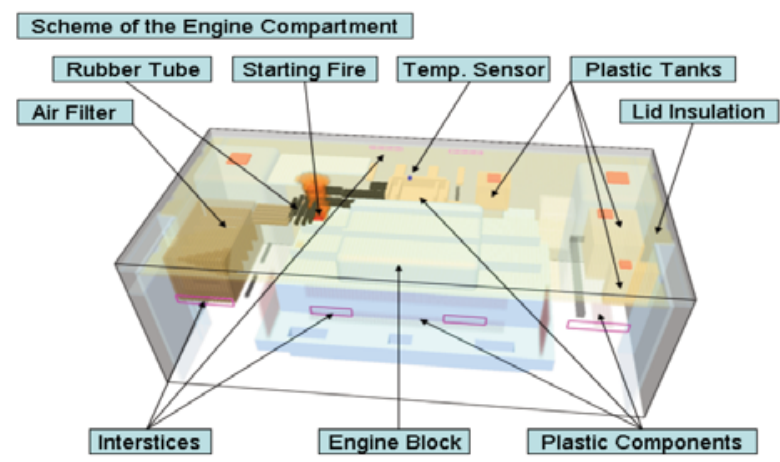

Fig. 1 Computer e model of automobile engine compartment

Proper determination of material properties (physical parameters describing burning properties of materials) in engine compartment was another essential task to be solved. There were four prevailing types of materials identified: aluminium alloy (metallic components), rubber (rubber tube), paper (air filter interior) and mixture of polyethylene (plastic components). Some material parameters for automobile varnish were estimated (e.g. the heat release rate per unit area) and some of them were derived from observa- tions (e.g. the ignition temperature was determined from recorded infra-red camera observations) during the full-scale fire experiment.

In the simulation, the fire ignition source (the small burning cloth placed on the engine block under the rubber tube) is represented by a burning surface with the dimensions of $4 \times 4 \mathrm{~cm}$ and total heat release rate set to $2.1 \mathrm{~kW}$ for the period of $60 \mathrm{~s}$ (see Fig. 1). Computational domain consists of two meshes including the interior of engine compartment as well as additional space above the engine compartment bonnet. The first mesh includes the interior of engine compartment and $3 \mathrm{~cm}$ space above the engine compartment bonnet, in order to show also the temperature distribution on the upper surface of the bonnet. The mesh boundary conditions are given by the material properties of bodywork (aluminium alloy). The mesh size was $132 \times 72 \times 53 \mathrm{~cm}$ with $1 \times 1 \times 1 \mathrm{~cm}$ resolution $(503,712$ cells total). The second mesh placed above the bonnet includes the space in which potential varnish fire occurs during the simulation. Its size is $172 \times 112 \times 80 \mathrm{~cm}$ with the resolution decreased to $2 \mathrm{~cm}(192,640$ additional cells). Each of two meshes was assigned to one CPU core. The simulation of $720 \mathrm{~s}$ of fire required 65 hours of CPU time at Intel Q9550, $2.83 \mathrm{GHz}$ CPU. More detailed comparison between full-scale experiment of the engine compartment fire of Audi 80 and computer simulation is described in [14].

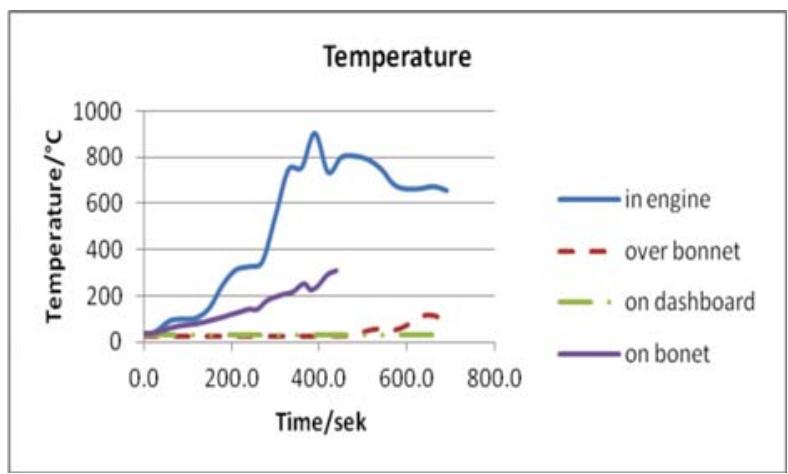

Fig. 2 Temperature in engine compartment obtained by full-scale experiment (IR)

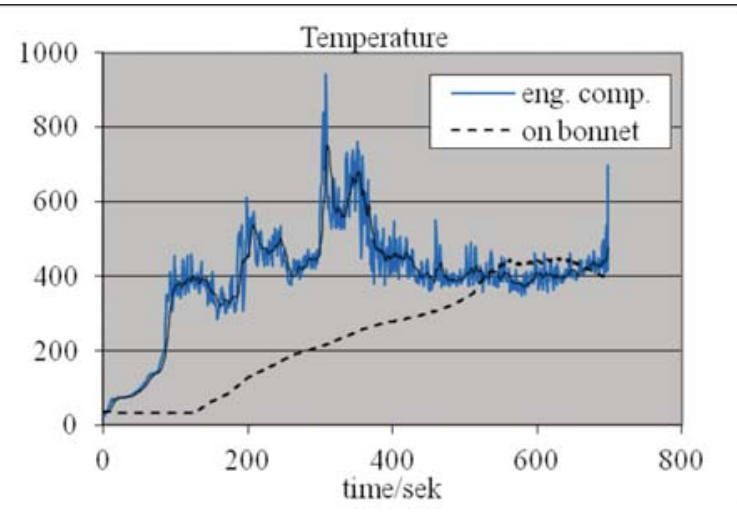

Fig. 3 Temperature in engine and on the bonnet obtained by FDS simulation 


\section{Computer simulation of additional types of car fires}

A simple case of the simulation of ignition of a near-standing automobile from burning vehicle is shown in Fig. 4. We used two identical automobiles of the same type as in the previous simulation.The first automobile represents a source of fire similar to that in the previous case. The distance between the automobiles was $60 \mathrm{~cm}$, what is in actual practice usually an upper border. Wind direction was chosen to accelerate the spread of flames from first to the second automobile. The size of computational domain was $510 \times 540 \times 210$ with $3 \mathrm{~cm}$ computational mesh resolution. The total number of cells was equal to 2142000 . The domain was split into four meshes which were assigned to four CPU cores of Inte Q9550 CPU, $2.83 \mathrm{GHz}$ CPU. The simulation in the $4^{\text {th }}$ minute of fire, a short time after ignition of the second car, is shown in Fig. 4. By this way we are able to simulate and analyze ignition of nearstanding automobile from burning vehicle depending on the distance between the automobiles in different conditions in open and closed area for miscellaneous car category.

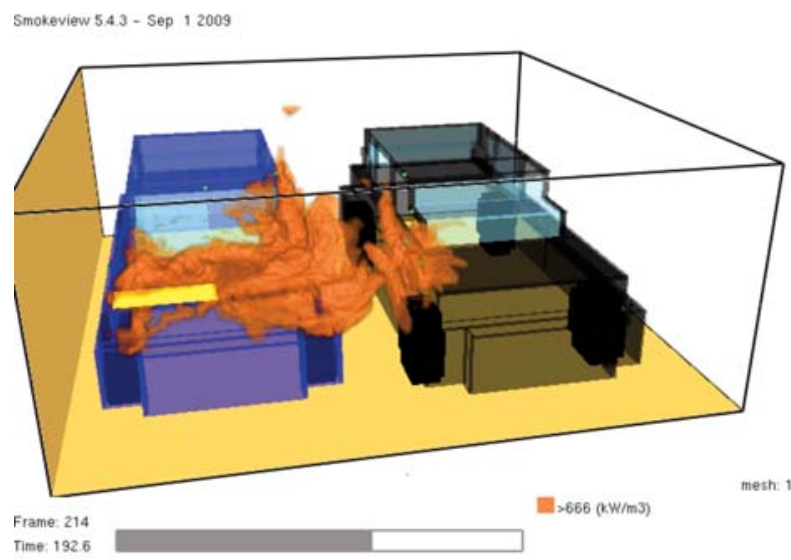

Fig. 4 Ignition of near standing automobile

Fires of automobile seating and/or luggage compartment are not very frequent. However, if they occur (e. g. as a result of arson),

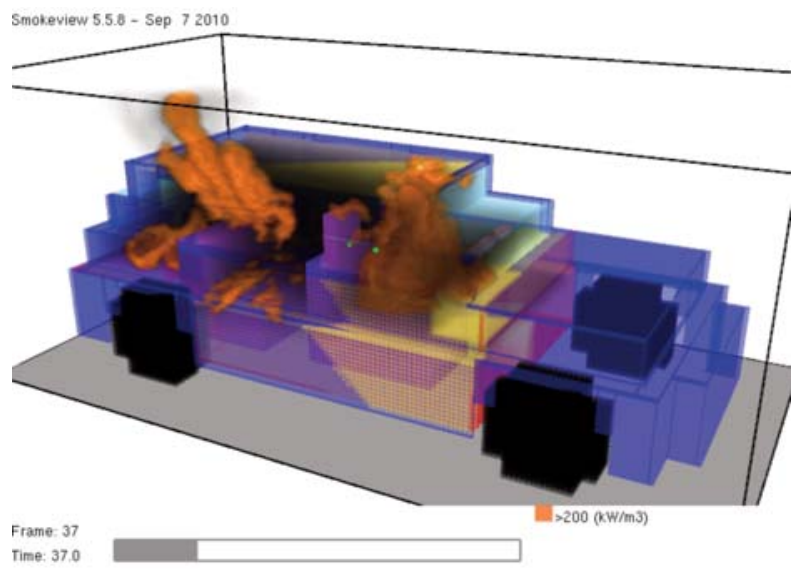

Fig. 5 Fire of automobile seating and luggage space they can be very dangerous particularly in urban conglomeration. Such fires can cause large damages especially in the case, when the interior of automobile is sufficiently supplied with oxygen during fire (for example, if it flows through a broken window or ajar door). Computer simulation of such a case is illustrated in Fig. 5.

In order to show the FDS simulation of automobile fire in tunnel, we constructed a model of road tunnel with dimensions 10 $\times 180 \times 7 \mathrm{~m}$ (width $\times$ length $\times$ height, $\mathrm{XYZ}$ ) with a simple ventilation system consisting of two fans located under the ceiling $50 \mathrm{~m}$ far from both tunnel ends. The Cartesian coordinate system for the tunnel was chosen as $\mathrm{X} \times \mathrm{Y} \times \mathrm{Z} \in[-5.0,5.0] \times[0.0$, $180.0] \times[-4.8,2.2]$ in meters. The mean gas temperature was measured by the top detectors $\mathrm{T} 1, \mathrm{~T} 2$ and $\mathrm{T} 3$ in the position [0.0, $20.2,1.8],[0.0,125.2,1.8]$ and $[0.0,160.2,1.8]$, respectively and by the bottom detectors $\mathrm{B} 1, \mathrm{~B} 2, \mathrm{~B} 3$ in the positions $[0.0,20.2$, $-3.1],[0.0,125.2,-3.1]$ and $[0.0,160.2,-3.1]$, respectively (see Fig. 6). The first wall position is exactly over the fire, while the second one is located about $30 \mathrm{~m}$ downstream the air blown by fans.

The fire was simulated by burning of combustible block with dimensions $[0.0,2.0] \times[97.0,100.0] \times[-3.8,-3.7]$ with the maximum heat release rate $1000 \mathrm{~kW}$. The initial temperature of air in the whole tunnel was set to $20^{\circ} \mathrm{C}$. The total simulation time was $150 \mathrm{~s}$ and the dynamics of fire and fans was simulated as follows. At the beginning, the fans started to blow the air with the velocity of $5 \mathrm{~m} / \mathrm{s}$ in Y-direction. At $40^{\text {th }}$ second, the fire started with linearly increasing power, so that the maximum intensity was achieved at the $45^{\text {th }}$ second and was not changed until the end of simulation. Simultaneously, the fans started to increase the air velocity linearly from the $50^{\text {th }}$ second onwards, whereby the final velocity of $20 \mathrm{~m} / \mathrm{s}$ was achieved at the $55^{\text {th }}$ second and was not changed afterwards.

Computations were performed in parallel using FDS, version 5.4.0 on the Woodcrest Cluster at Regionales Rechenzentrum Erlangen, Erlangen - Nuernberg University (Germany). This cluster consists of 217 computational nodes, each with two Xenon 5160 Woodcrest chips ( 4 cores organized in 2 dual cores) running at 3.0 GHz. Each dual core contains $4 \mathrm{MB}$ shared Level 2 cache, 8 GB of RAM and $160 \mathrm{~GB}$ of local scratch disk. The Infiniband interconnection network has the bandwidth of $10 \mathrm{Gbit} / \mathrm{s}$ per link and direction.

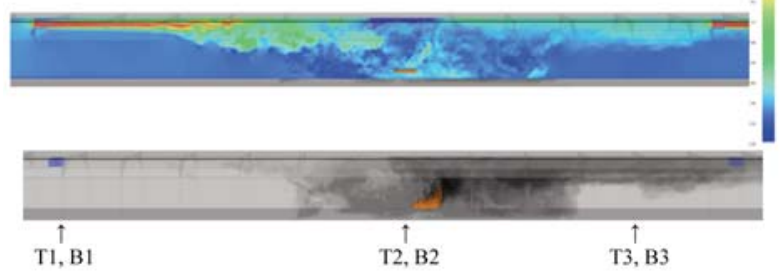

Fig. 6 Distribution of gas velocity (top) and smoke movement (bottom) in the tunnel at the 80th second 


\section{Conclusion}

In this paper, it is shown how the computer simulation can be useful for visualization and analysis of automobile fire processes. Four types of automobile fire simulations are presented. Firstly, the FDS simulation of automobile engine compartment fire, validated by results of the full-scale fire experiment in the testing facilities of Fire Protection College of the Ministry of Interior of Slovak Republic in Povazsky Chlmec, is described. A good similarity between the simulation results and experimentally measured variables was confirmed. Next, the FDS simulations of automobile fires ignited in seating and luggage compartments, as well as the ignition of near standing automobile and the automobile fire in tunnel are illustrated. The simulation results will be also validated by the data obtained from the full-scale fire experiments. The presented simulation results show the great potential to model even relatively very complicated fire scenarios in very different environments.

In [15], some basic objectives how to achieve higher level security from legislative and civil point of view in Slovakia were briefly formulated. We would like to remind that the increase of security and safety in each economically and technically developed country includes also a number of technical and tactical issues which crisis management must take into account $[16,17]$.

Acknowledgments. The authors would like to thank to . col. Ing. Jaroslav Flachbart, PhD., the director of Fire Protection College of the Ministry of Interior of Slovak Republic in Zilina for his kind help during the fire experiments. This work was partially supported by Slovak Scientific Research Agencies APVV (project APVV-0532-07) and VEGA (project VEGA 2/0216/10).

\section{References}

[1] BABRAUSKAS, V., WILLIAMSON, R. B.: Post-flashover Compartment Fires: Basis of a Theoretical Model. Fire and Materials, Vol. 2, 1978, pp. 39-53.

[2] PETTERSSON, O., MAGNUSSON, S. E., THOR, J.: Fire Engineering Design of Steel Structures. Swedish Institute of Steel Construction, Stockholm, Publication 50, 1976.

[3] BANJAC, M. J., NIKOLIC, B. M.: Computational Study of Smoke Flow Control in Garage Fires and Optimization of the Ventilation System. Thermal Science, Vol. 13, No. 1, pp.69-78, 2009.

[4] ZHAO, B., KRUPPA, J.: Structural Behaviour of an Open Car Park under Real Fire Scenarios, Fire and Materials, Vol. 28, 2004, pp. $269-280$.

[5] ZHANG, X. G., GUO, Y. C., CHAN, C. K., LIN, W. U.: Numerical Simulations on Fire Spread and Smoke Movement in a Underground Car park. Building and Environment, Vol. 42, 2007, pp. 3466-3475.

[6] VIEGAS, J. C.: The Use of Impulse Ventilation for Smoke Control in Underground Car Parks. Tunnelling and Underground Space Technology, Vol. 25, 2010, pp. 42-53.

[7] GAO, P. T., LIU, S. L., CHOW, W. K., FONG, N. K.: Large Eddy Simulations for Studying Tunnel Smoke Ventilation. Tunnelling and Underground Space Technology, Vol. 19, 2004, pp. 577-586.

[8] HU, L. H., HUO, R., PENG, W., CHOW, W. K., YANG, R. X.: On the Maximum Smoke Temperature under the Ceiling in Tunnel Fires. Tunnelling and Underground Space Technology, Vol. 21, 2006, pp. 650-655.

[9] LI, J. S. M., CHOW, W. K.: Numerical Studies on Performance Evaluation of Tunnel Ventilation Safety System. Tunnelling and Underground Space Technology, Vol. 18, 2003, pp. 435-452.

[10] MIGOYA, E., CRESPO, A., GARCIA, J., HERNANDEZ, J.: A Simplified Model of Fires in Road Tunnels. Comparison with Three-dimensional Models and Full-scale Measurements, Tunnelling and Underground Space Technology, Vol. 24, 2010, pp.37-52.

[11] Mc GRATTAN, K., et al.: Fire Dynamics Simulator, Technical reference guide. National Institute of Standards and Technology, Version 5.3, 2009, USA.

[12] LIN, C. H., FERNG, Y. M., HSU, W. S.: Investigating the Effect of Computational Grid Sizes on Predicted Characteristics of Thermal Radiation for a Fire. Applied Thermal Engineering, Vol. 29, 2009, pp. 2243-2250.

[13] FERNG, Y. M., LIN, C. H.: Investigating of Appropriate Computational Mesh Size and Solid Angle for CFD Simulating the Characteristics of Pool Fires with Experiments Assessment. Nuclear Engineering and Design, Vol. 240, 2010, pp. 816-822.

[14] WEISENPACHER, P., GLASA, J., HALADA, L.: Computer Simulation of Automobile Engine Compartment Fire, Proc. of the Int. Congress on "Combustion and Fire Dynamics", Santander, 2010, pp. 257-269.

[15] SIMAK, L.: Increasing the Security Level in the Slovak Republic, Communications - Scientific Letters of the University of Zilina, Vol. 2, 2008, pp. 67-71,

[16] JANACEK, J., SIBILA, M.: Optimal Evacuation Plan Designed with IP/Solver. Communications - Scientific Letters of the University of Zilina, Vol. 3, 2009, pp. 29-35.

[17] HRBECEK, J., JANOTA, A.: Improvement of Road Tunnel Ventilation through Predictive Control, Communications - Scientific Letters of the University of Zilina, Vol. 2, 2008, pp. 15-19. 